\title{
Peritumoral cystic meningioma: A report of two cases and review of the literature
}

\author{
PENGFEI WANG ${ }^{1 *}$, SONG HAN $^{1 *}$, NING LIU $^{1}$, CHUNJIANG YU $^{1}$, XUELING QI $^{2}$, \\ MINGWANG ZHU ${ }^{3}$, XIANGQIAN ZHANG ${ }^{1}$, LI WANG $^{1}$ and CHANGXIANG YAN ${ }^{1}$ \\ Departments of ${ }^{1}$ Neurosurgery, ${ }^{2}$ Pathology and ${ }^{3}$ Radiation, Beijing Sanbo Brain Hospital, \\ Capital Medical University, Beijing 100093, P.R. China
}

Received November 16, 2014; Accepted October 27, 2015

DOI: $10.3892 /$ etm.2016.2999

\begin{abstract}
The present study reported two cases of cystic meningioma. The clinical manifestations, magnetic resonance imaging (MRI) scan and histological aspects of the lesion and the associated cyst were examined. The classification of cystic meningioma was also discussed. The present study focused on the formation, diagnosis and management of the peritumoral cystic meningioma, and aimed to clarify certain contradictions in the literature concerning the formation of the peritumoral cyst meningioma: MRI alone is inadequate to determine the type of cystic meningioma, or to identify neoplastic cells on the cystic wall. In conclusion, surgical removal of the entire cyst is recommended in peritumoral cyst meningioma.
\end{abstract}

\section{Introduction}

Cystic meningioma is a relatively rare condition with incidence rates ranging between 1.7 and 10\% worldwide (1-5). While meningiomas are considered more likely to appear in females, no substantial difference was identified in the incidence rates between the two genders, according to an analysis of 166 cases of cystic meningioma (2). The clinical manifestation of cystic meningioma include, but are not limited to: Headache, seizure, dizziness, personality changes and motor disturbance (1-10). Surgical removal of the entire tumor and its cyst remains the predominant treatment for cystic meningioma (1-10). Cystic meningioma may pose a diagnostic dilemma preoperatively, since it is occasionally difficult to differentiate among glioma, hemangioblastoma, craniopharyngioma and metastatic brain

Correspondence to: Dr Changxiang Yan, Department of Neurosurgery, Beijing Sanbo Brain Hospital, Capital Medical University, 50 Xiang Shan Yi Ke Song Road, Haidian, Beijing 100093, P.R. China

E-mail: yancx65828@sina.com

${ }^{*}$ Contributed equally

Key words: cystic meningioma, peritumoral cystic meningioma, magnetic resonance imaging, pathology tumor (6-10). The accuracy of the diagnosis of cystic meningioma based on the results of magnetic resonance imaging (MRI) has been reported to be $78 \%$ preoperatively (4). The present study reported two cases of benign meningioma with a large cyst, which presented with a peritumoral cyst with neoplastic cells on the cystic wall.

\section{Case report}

Case 1. A 57-year-old male patient presented at the Beijing Sanbo Brain Hospital (Beijing, China) on November $2^{\text {nd }} 2013$ with a left-sided hemiparesis that persisted for 11 months, which was accompanied by a headache lasting 3 days prior to admission. A neurological examination was completed which revealed clouding of consciousness, speech disturbance, decreased left-side distal muscle strength (grade 0) and proximal muscle strength (grade III), according to the WHO classification (11). A contrast-enhanced MRI scan (Fig. 1A and B) was performed using $10 \mathrm{ml}$ gadolinium-diethylenetriaminepentaacetic acid (Bayer AG, Leverkusen Germany) and reported according to previous studies $(12,13)$. The MRI scan revealed a large, homogeneously enhancing lesion with multiple cysts with wall enhancement in the right hemisphere. The midline was found to be markedly shifted and the left lateral ventricle was dilated. The solid tumor parts and the cystic wall were also found to be enhanced. The tumor measured $5.7 \times 6.2 \times 7.0 \mathrm{~cm}$ and was surrounded by edema (Fig. 1C). A 2-deoxy-2-[18F]-fluoro-Dglucose positron emission tomography/computed tomography scan revealed a slightly increased metabolism in the solid components of the tumor and the maximum standardized uptake value was 2.4 (Fig. 1D). Based on the clinical manifestations and the imaging results, glioma was diagnosed and surgery was performed to remove the tumor.

During surgery, a tumor was found within the cyst, along with multiple other cysts within the tumor. Histological examination demonstrated that the tumor was of the angiomatous type with hyaline degeneration of the vessel wall (Fig. 2). Biopsy of the cystic wall revealed gliosis and presence of tumor cells (Fig. 2). This configuration was classified as a peritumoral cystic meningioma with neoplastic cells on the cystic wall.

The patient did not receive further medical treatment following the operation. An MRI scan was performed 
A

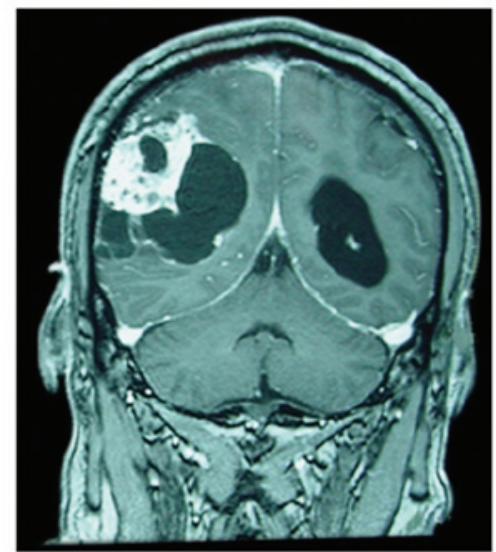

C

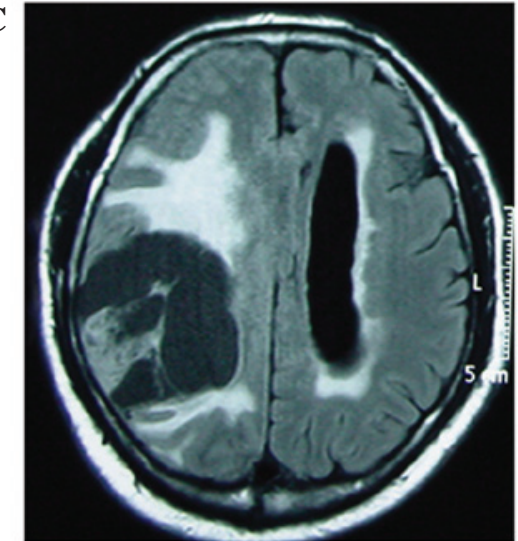

B

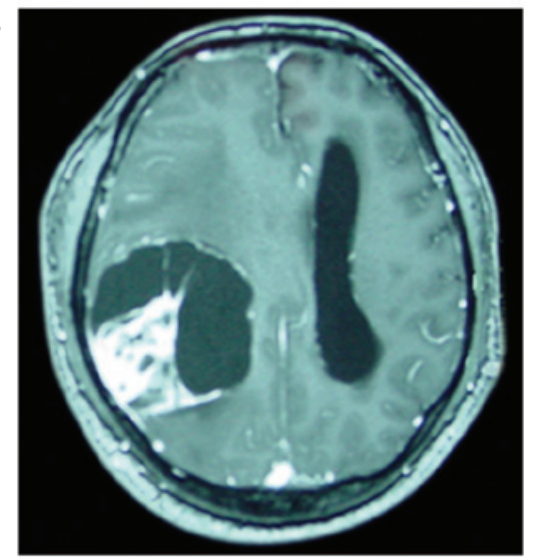

D

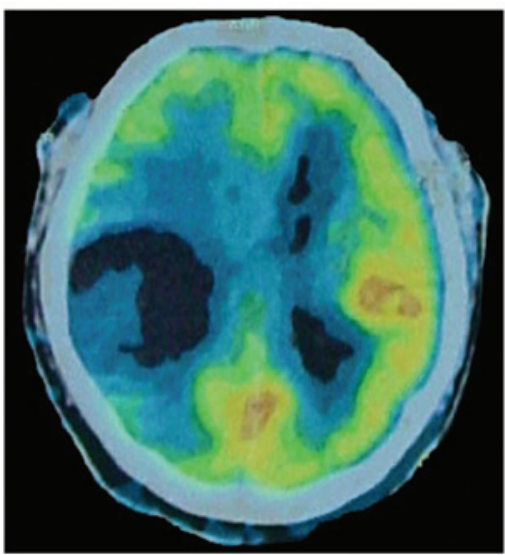

Figure 1. Case 1: (A) Axial and (B) coronal T1-weighted contrast-enhanced MRI scans. (C) Axial fluid-attenuated inversion recovery MRI and (D) positron emission tomography/computed tomography scans. MRI, magnetic resonance imaging.

A

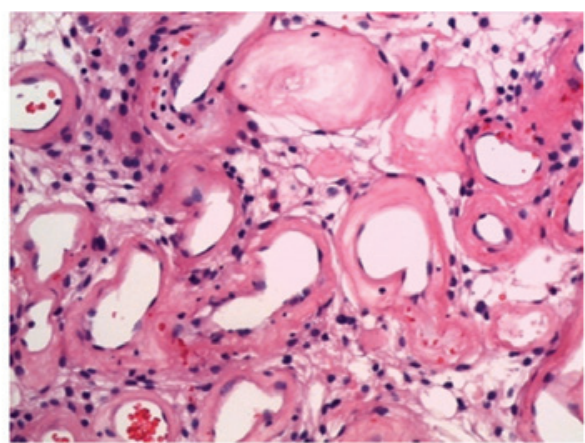

B

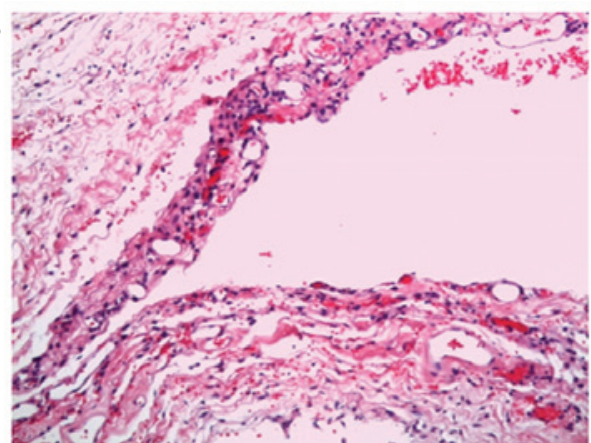

Figure 2. Case 1: H\&E staining of the solid tumor. (A) Microscopic examination of the solid tumor demonstrated an angiomatous pattern with hyaline degeneration of the vessel wall (original magnification, x200). (B) The cystic wall contained neoplastic cells and fibrous tissue (original magnification, x100). H\&E, hematoxylin and eosin.

3 months postoperatively without any evidence of tumor. The patient was followed up on November 1st 2015 via telephone, he is in good health with the exception that his left finger is not as flexible as it previously has been. Written informed consent was obtained from the patient.

Case 2. A 29-year old male patient presented at the Beijing Sanbo Brain Hospital on November 12 2013 with urinary incontinence and memory decline for 2 months. A neurological examination showed no deficits. MRI scans revealed an enhancing frontal solid mass with multiple well-circumscribed peritumoral cysts with an irregularly enhancing wall
(Fig. 3). Based on the clinical manifestations and the imaging results, glioma was diagnosed and surgery was performed to remove the tumor.

During surgery, the tumor nodule was found to be surrounded by several closely applied cysts and multiple biopsies of the cystic wall revealed the presence of tumor cells. Histopathological analysis showed that the tumor was a meningothelial meningioma that exhibited partly active proliferation, and was classified as a grade II tumor according to the World Health Organization classification (11). Necrosis, calcification, mitosis and reactive gliosis were also observed. Biopsy of the cystic wall demonstrated that it was composed 
A

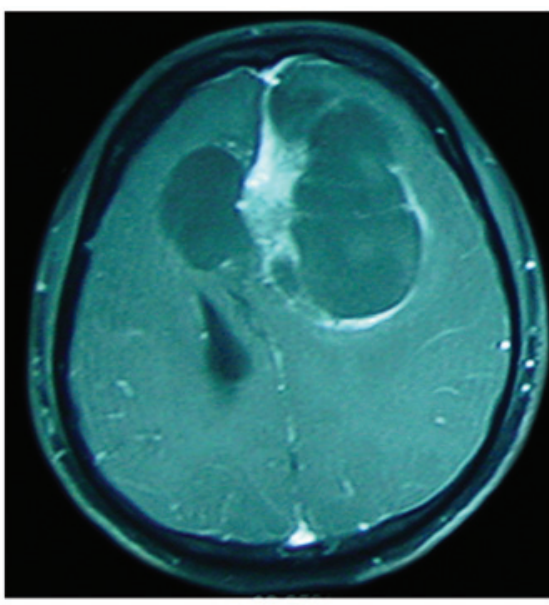

B

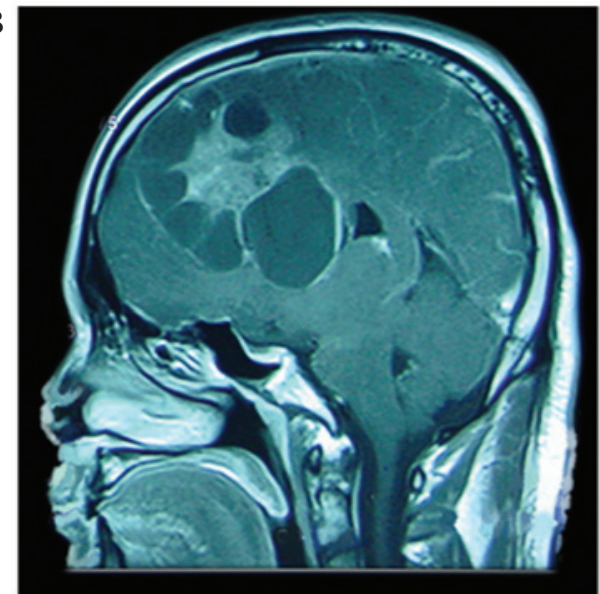

Figure 3. Case 2: (A) Axial and (B) sagittal T1-weighted contrast-enhanced magnetic resonance imaging scans

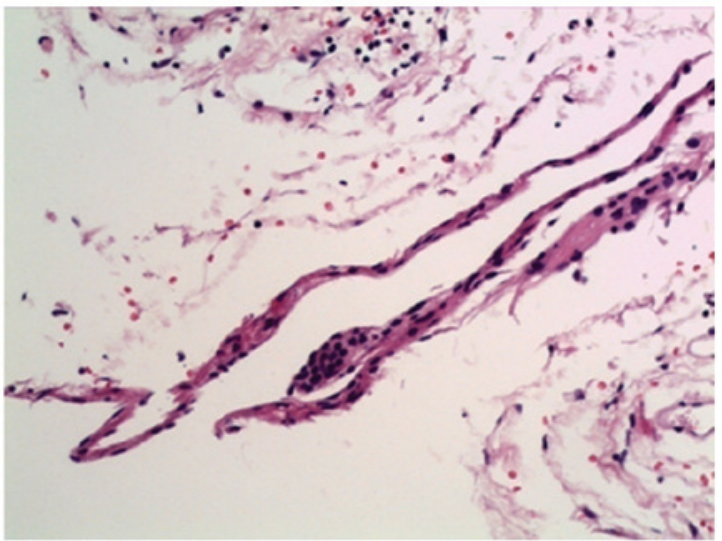

Figure 4. Case 2: Histopathological examination following staining with hematoxylin and eosin demonstrated that the cyst wall contained meningioma cells and fibrous tissue (magnification, x200).

of fibrous tissue and tumor cell nests (Fig. 4). The final diagnosis was peritumoral cystic meningioma with tumor invasion on the cystic wall.

The patient received one dose of radiotherapy following the operation. An MRI was performed in July 2015 and no tumors were detected. The patient was followed up on November 1st 2015 via telephone, he is in good health and is able to work and exercise as before. Written informed consent was obtained from the patient.

\section{Discussion}

The most common site for the appearance of cystic meningiomas is the cerebral convexity, particularly the frontal and parietal lobes, while the cerebral falx is the second most frequent location (2). Furthermore, cystic meningiomas can also be identified in the cerebellopontine angle, olfactory groove, suprasellar, falx and ventricle, olfactory groove trigeminal nerve or optic nerve $(3,5,6,14-19)$. The most frequent histological subtype has been found to be the meningothelial subtype $(2,5,20)$. Notably, atypical meningiomas have the tendency to form a cyst, as opposed to other subtypes of meningioma $(17,20)$.
Rengachary et al (21) recognized two types of cystic meningioma, including the intratumoral and peritumoral cystic meningiomas. Nauta et al (22) subdivided these two types according to the association between the tumor, associated cysts and the surrounding brains, as follows: Type I, which has a central cyst; type II, which has a peripheral intratumoral cyst; type III, which has a peritumoral cyst that lies within the adjacent brain region; and type IV, which has a peritumoral cyst at the interface of the tumor and brain. Worthington et al (23) then added a fifth type to the Nauta classification: Type V, in which the cysts enclosed the tumor nodule with the neoplastic cells on the cystic wall. Weber et al (24) subdivided the peritumoral cystic meningioma according to the presence of tumor invasion in the cystic wall.

The underlying mechanism of cyst formation remains unclear. Intratumoral cysts (Nauta types I and II) are likely to be caused by microcystic degeneration, ischemic necrosis or hemorrhage within the tumor $(2,23,25)$. In addition, transudation or secretory changes within the meningioma may also lead to the formation of cysts (25); therefore, the cystic wall of intratumoral cysts (Nauta types I and II) contains neoplastic cells and its immediate removal is required $(3,26,27)$. The formation of peritumoral cysts (Nauta type III) may be due to reactive gliosis toward the meningioma or the evolution of cerebral edema to form peritumoral cystic cavities $(22,23)$. Nauta type IV cysts may be a result of the widening of the subarachnoid spaces and the trapped cerebrospinal fluid around the tumor $(21-23,25,28)$. A previous study reported a strong expression of aquaporin 1 in a case of peritumoral cystic meningioma (29). Consequently, the wall of peritumoral cysts is likely to be free from tumor cells and its excision is not required $(7,26)$. However, the cystic wall of peritumoral cystic meningioma has frequently been reported to be composed of neoplastic cells $(3,19,21,24,30)$. The presence of tumor cells on the cystic wall was believed to result from the spreading of the xanthochromic fluid in the peritumoral cyst to the wall $(3,17,24)$. This point of view, however, may provide an insufficient explanation based on the following two reasons: A study showed that despite the existence of meningioma cells (atypical) in the xanthochromic fluid, the cystic wall was not invaded by tumor cells (24). In addition, other studies found that the cystic wall was composed of neoplastic cells 
(meningothelial type, benign) without the presence of tumor cells in the xanthochromic fluid $(21,23,24)$. However, in chordoid or papillary meningiomas, which take on aggressive metastatic properties, the cystic wall has frequently been shown to be composed of tumor cells $(19,30,31)$. This is likely to occur due to the subarachnoid dissemination of tumor cells $(19,32)$. The presence of meningioma cells in the cystic fluid is not necessarily an indicator of tumor invasion in the wall. The formation of peritumoral cystic meningioma with neoplastic cells on the wall has been suggested to result from active secretion by the functional tumor cells and responsive glial proliferation, as well as the possible involvement of necrotic degeneration (3,23). Degeneration, necrosis, calcification and mitosis were also observed in the present two cases. The mechanism underlying the formation of peritumoral cyst meningioma with neoplastic cells on the wall involves degeneration, necrosis, responsive glial proliferation, spreading of neoplastic cells in the fluid or a combination of these (11).

MRI remains the optimal diagnostic means for the diagnosis of cystic meningioma, but it is not sufficient to determine the particular tumor type (27). Only microsurgical inspection and histological examination can lead to the final diagnosis of the type of cystic meningioma (27). Combined MRI and diffusion-weighted imaging (DWI) may be efficient in diagnosing the type of cystic meningioma $(4,33)$; however, as glioma was the initial diagnosis in the present study, DWI was not utilized. Chen et al (33) reported that the apparent diffusion coefficient ratio of intratumoral cystic meningioma obtained using DWI was lower compared with that of peritumoral cystic meningioma, based on only three cases. Contrast-enhanced MRI may assist surgeons in determining whether neoplastic cells are present in the cystic wall; however, the wall could be enhanced despite the absence of tumor cells (34). Furthermore, biopsy was found to detect tumor cells on the cystic wall, even in cases where these were not detected by contrast-enhanced MRI $(3,27)$. It is therefore not accurate to support the absence of tumor cells based merely on the negative results of contrast-enhanced MRI $(20,27,35)$.

In conclusion, combined MRI and DWI may provide valuable assistance in the diagnosis of cystic meningioma and its various types. The diagnosis value of PET/CT in cystic meningioma requires further investigation and previous studies have demonstrated that DWI may be superior at distinguishing between the various types of meningioma $(4,30)$. Two cases of cystic meningioma were evaluated in the present case report and microsurgical inspection, multiple biopsies and frozen sections were sufficient to evaluate the presence or absence of tumor cells on the cystic wall $(17,24,27)$. However, the diagnostic accuracy of the presence of tumor cells on the cyst wall was not $100 \%$; therefore, biopsy remains the most accurate and reliable treatment option, as compared with imaging methods, and we recommend whole surgical removal of the cyst.

\section{References}

1. Zee CS, Chen T, Hinton DR, Tan M, Segall HD and Apuzzo ML: Magnetic resonance imaging of cystic meningiomas and its surgical implications. Neurosurgery 36: 482-488, 1995.

2. Fortuna A, Ferrante L, Acqui M, Guglielmi G and Mastronardi L: Cystic meningiomas. Acta Neurochir (Wien) 90: 23-30, 1988.
3. Jung TY, Jung S, Shin SR, Moon KS, Kim IY, Park SJ, Kang SS and Kim SH: Clinical and histopathological analysis of cystic meningiomas. J Clin Neurosci 12: 651-655, 2005.

4. Zhang D, Hu LB, Zhen JW, Zou LG, Feng XY, Wang WX and Wen L: MRI findings of intracranial cystic meningiomas. Clin Radiol 64: 792-800, 2009.

5. Sridhar K, Ravi R, Ramamurthi B and Vasudevan MC: Cystic meningiomas. Surg Neurol 43: 235-239, 1995.

6. Goyal A, Singh AK, Gupta V, Singh D, Tatke M and Kumar S: Suprasellar cystic meningioma: Unusual presentation and review of the literature. J Clin Neurosci 9: 702-704, 2002.

7. Ueno Y, Tanaka A, Nakayama Y and Nomoto Y: Intracerebral cyst associated with meningioma. Clin Neurol Neurosurg 101: 271-274, 1999.

8. Guan TK, Pancharatnam D, Chandran H, Hooi TK, Kumar G and Ganesan D: Infratentorial benign cystic meningioma mimicking a hemangioblastoma radiologically and a pilocytic astrocytoma intraoperatively: A case report. J Med Case Rep 7: 87, 2013.

9. Yamada SM, Fujimoto Y, Kawanishi Y and Shimizu K: A cystic meningioma misdiagnosed as malignant glioma by radiologic and intraoperative histological examinations. Brain Tumor Pathol 27: $111-115,2010$.

10. Rishi A, Black KS, Woldenberg RW, Overby CM, Eisenberg MB and Li JY: Microcystic meningioma presenting as a cystic lesion with an enhancing mural nodule in elderly women: Report of three cases. Brain Tumor Pathol 28: 335-339, 2011.

11. Louis DN, Ohgaki H, Wiestler OD, Cavenee WK, Burger PC, Jouvet A, Scheithauer BW and Kleihues P: The 2007 WHO classification of tumours of the central nervous system. Acta Neuropathol 114: 97-109, 2007.

12. Xia L, Zhang $\mathrm{H}$, Yu C, Zhang M, Ren M, Qu Y, Wang H, Zhu M, Zhao D, Qi X and Yao K: Fluid-fluid level in cystic vestibular schwannoma: A predictor of peritumoral adhesion. J Neurosurg 120: 197-206, 2014.

13. Wang YQ, Fan T, Zhao XG, Liang C, Qi XL and Li JY: Pituitary carcinoma with intraspinal metastasis: Report of two cases and review of the literature. Int J Clin Exp Pathol 8: 9712-9717, 2015.

14. Deb P, Sahani H, Bhatoe HS and Srinivas V: Intraventricular cystic meningioma. J Cancer Res Ther 6: 218-220, 2010.

15. Obeng K, Rumboldt Z, Tuite G, Welsh CT, Patel S and Spampinato MV: Atypical cystic meningioma of the trigeminal nerve in a pediatric patient. AJNR Am J Neuroradiol 29: 398-399, 2008.

16. Fujimoto Y, Kato A, Taniguchi M, Maruno M and Yoshimine T: Meningioma arising from the trigeminal nerve: A case report and literature review. J Neurooncol 68: 185-187, 2004.

17. Hu SL, Li F, Hu R, Cui G, Meng H and Feng H: Atypical histopathologic type of cystic meningioma. Acta Neurochir (Wien) 152 . 105-109, 2010.

18. Rosca TI, Carstocea BD, Vlãdescu TG, St Tihoan C and Gherghescu GG: Cystic optic nerve sheath meningioma. J Neuroophthalmol 26: 121-122, 2006.

19. Zhi L, Bing L, Yang L, Bo-ning L and Quan H: Cystic papillary meningioma with subarachnoid dissemination: A case report and review of the literature. Pathol Res Pract 205: 582-587, 2009.

20. Senbokuya N, Asahara T, Uchida M, Yagishita T and Naganuma H: Atypical meningioma with large cyst. Case report. Neurol Med Chir (Tokyo) 46: 147-151, 2006.

21. Rengachary S, Batnitzky S, Kepes JJ, Morantz RA, O'Boynick P and Watanabe I: Cystic lesions associated with intracranial meningiomas. Neurosurgery 4: 107-114, 1979.

22. Nauta HJ, Tucker WS, Horsey WJ, Bilbao JM and Gonsalves C: Xanthochromic cysts associated with meningioma. J Neurol Neurosurg Psychiatry 42: 529-535, 1979.

23. Worthington C, Caron JL, Melanson D and Leblanc R: Meningioma cysts. Neurology 35: 1720-1724, 1985.

24. Weber J, Gassel AM, Hoch A, Kilisek L and Spring A: Intraoperative management of cystic meningiomas. Neurosurg Rev 26: 62-66, 2003.

25. Odake G: Cystic meningioma: Report of three patients. Neurosurgery 30: 935-940, 1992.

26. Zhao X, Sun JL, Wang ZG, Zhang TG, Wang CW and Ji Y: Clinical analysis for an unusual large cystic meningioma: Case report and review of the literature. Clin Neurol Neurosurg 110: 605-608, 2008.

27. Ferrante L, Acqui M, Lunardi P, Qasho R and Fortuna A: MRI in the diagnosis of cystic meningiomas: Surgical implications. Acta Neurochir (Wien) 139: 8-11, 1997.

28. Sigel RM and Messina AV: Computed tomography; the anatomic basis of the zone of diminished density surrounding meningiomas. AJR Am J Roentgenol 127: 139-141, 1976. 
29. Marton E, Feletti A, Basaldella L, Dei Tos AP, Bendini M and Longatti P: Atypical cystic meningioma overexpressing AQP1 in early infancy: Case report with literature review. Acta Paediatr 97: 1145-1149, 2008

30. Zhao SL,Li Y,Tian XY,LiZ, Huang Q and Li B: Intraparenchymal cystic chordoid meningioma: A case report and review of the literature. Neuropathology 31: 648-653, 2011

31. Enam SA, Abdulrauf S, Mehta B, Malik GM and Mahmood A: Metastasis in meningioma. Acta Neurochir (Wien) 138: 1172-1177, discussion 1177-1178, 1996.

32. Wakabayashi K, Suzuki N, Mori F, Kamada M and Hatanaka M Rhabdoid cystic papillary meningioma with diffuse subarachnoid dissemination. Acta Neuropathol 110: 196-198, 2005.
33. Chen TY, Lai PH, Ho JT, Wang JS, Chen WL, Pan HB, Wu MT, Chen C, Liang HL and Yang CF: Magnetic resonance imaging and diffusion-weighted images of cystic meningioma: Correlating with histopathology. Clin Imaging 28: 10-19, 2004.

34. Arai M, Kashihara K and Kaizaki Y: Enhancing gliotic cyst wall with microvascular proliferation adjacent to a meningioma. J Clin Neurosci 13: 136-139, 2006.

35. Demir MK, Müslüman M, Kilicoglu G, Hakan T and Aker FV: Imaging features of unusual intracranial cystic meningiomas. Can Assoc Radiol J 58: 109-115, 2007. 